\title{
Time from first detectable PSA following radical prostatectomy to biochemical recurrence: A competing risk analysis
}

\author{
Leonora de Boo, MD; ${ }^{*}$ Melania Pintilie, PhD; ${ }^{\dagger}$ Paul Yip; Jack Baniel, MD; ${ }^{*}$ Neil Fleshner, MD, FRCSC; \\ David Margel, MD, PhD
}

*Division of Urology, Department of Surgical Oncology,University Health Network, Toronto, ON and Erasmus MC-beurs, Erasmus University Rotterdam, Netherlands; ${ }^{\dagger}$ Clinical Study Coordination and Biostatistics, University Health Network, Toronto, ON; §Laboratory Medicine Program, University Health Network, Toronto, ON; *Division of Urology, Rabin Medical Center and Sackler Faculty of Medicine, Tel Aviv University, Tel Aviv, Israel; „Division of Urology, Department of Surgical Oncology, University Health Network, Toronto, ON; £Division of Urology and Davidoff Cancer Center, Rabin Medical Center, Beilinson Hospital, Israel

Cite as: Can Urol Assoc J 2015;9(1-2):E14-21. http://dx.doi.org/10.5489/cuaj.2147

Published online January 12, 2015.

\section{Abstract}

Introduction: In this study, we estimated the time from first detectable prostate-specific antigen (PSA) following radical prostatectomy (RP) to commonly used definitions of biochemical recurrence (BCR). We also identified the predictors of time to BCR.

Methods: We identified subjects who underwent a RP and had an undetectable PSA after surgery followed by at least 1 detectable PSA between 2000 and 2011. The primary outcome was time to $B C R$ (PSA $\geq 0.2$ and successive PSA $\geq 0.2$ ) and prediction of the rate of PSA rise. Outcomes were calculated using a competing risk analysis, with univariable and multivariable Fine and Grey models. We employed a mixed effect model to test clinical predictors that are associated with the rate of PSA rise.

Results: The cohort included 376 patients. The median follow-up from surgery was 60.5 months (interquartile range [IQR] 40.891.5) and from detectable PSA was 18 months (IQR 11-32). Only $45.74 \%(\mathrm{n}=172)$ had PSA values $\geq 0.2 \mathrm{ng} / \mathrm{mL}$, while $15.16 \%$ $(\mathrm{n}=57)$ reached the PSA level of $\geq 0.4 \mathrm{ng} / \mathrm{mL}$ and rising. On multivariable analysis, the values of the first detectable PSA and pathologic Gleason grade 8 or higher were consistently independent predictors of time to BCR. In the mixed effect model rate, the PSA rise was associated with time from surgery to first detectable PSA, Gleason score, and prostate volume. The main limitation of this study is the large proportion of patients that received treatment without reaching BCR. It is plausible that shorter estimated median times would occur at a centre that does not use salvage therapy at such an early state.

Conclusion: The time from first detectable PSA to BCR may be lengthy. Our analyses of the predictors of the rate of PSA rise can help determine a personalized approach for patients with a detectable PSA after surgery.

\section{Introduction}

Radical prostatectomy (RP) is the surgical treatment of choice for clinically localized prostate cancer. Prostatespecific antigen (PSA) monitoring is the primary tool for detecting relapse of cancer after RP. About $27 \%$ to $53 \%$ of men undergoing RP will have a detectable serum PSA within 10 years of surgery. ${ }^{1-5}$ Although biochemical recurrence $(B C R)$ is an ominous finding and is associated with an increased risk of progression to metastatic disease, ${ }^{6}$ it precedes clinical recurrence by many years. ${ }^{7-9}$ Furthermore, many patients with BCR will never progress.

Currently, level 1 evidence suggests that adjuvant radiation should be administered to patients with high-risk prostate cancer following RP. ${ }^{10-13}$ However, adjuvant radiation has not been compared to early salvage radiation. ${ }^{14-16}$ Therefore, most urologists would rather use early salvage therapy, most commonly offered after reaching BCR rather than after the first detectable PSA. ${ }^{17}$ To the best of our knowledge, the time frame from the first detectable PSA to BCR has not been explored.

In this study we used the 4 most accepted definitions for BCR: (1) PSA $\geq 0.2 \mathrm{ng} / \mathrm{mL}$; (2) an initial PSA of $\geq 0.2 \mathrm{ng} / \mathrm{mL}$ and successive PSA $\geq 0.2 \mathrm{ng} / \mathrm{mL}$; (3) PSA $\geq 0.4 \mathrm{ng} / \mathrm{mL}$; and (4) $P S A \geq 0.4 \mathrm{ng} / \mathrm{mL}$ and rising. We examined the natural history of the first detectable PSA and determined the associated predictors of reaching BCR. ${ }^{6,17-20}$ Since physicians differ in the exact time and PSA level for which they offer salvage radiation treatment, we also provide additional analysis to predict the rate of PSA rise after the first detectable PSA.

\section{Methods}

We used a retrospective cohort design to test the natural history of patients with a detectable PSA following RP. Our 
objective was to report the time from the first detectable PSA to 4 commonly used definitions of BCR and to identify predictors of time to BCR. The study was approved by the institutional review board at the Princess Margaret Cancer Centre, University Health Network, Toronto, Canada.

Our study included patients who underwent RP for clinically localized prostate cancer between the January 1, 2000 and March 1, 2011. All subjects had to have an undetectable PSA after surgery followed by at least 1 detectable PSA. The cohort entry date was defined as the date of the first detectable PSA. We followed up eligible individuals until they experienced an event (4 different definitions of BCR), or among those who did not reach BCR, until last follow-up visit, whichever came first. Patients treated with salvage radiation or androgen-deprivation therapy (ADT) before reaching our definitions of BCR were not at risk for biochemical failure. Thus these patients experienced a competing event at the date of salvage treatment initiation. We excluded patients receiving any form of neoadjuvant or adjuvant therapy.

RP specimens were examined by specialist genitourinary pathologists at our institution. Following surgery, serum PSA levels were measured every 3 months for the first year, biannually in years 2 and 5, and annually thereafter. PSA assays used at our institution were AxSYM Total PSA assay (Abbott Diagnostics) before November 21, 2006, and the ARCHITECT Total PSA assay (Abbott Diagnostics) thereafter with a $0.04 \mathrm{ng} / \mathrm{mL}$ lower detection limit for both assays.

Our primary analysis used the most commonly used BCR definition: initial PSA $\geq 0.2 \mathrm{ng} / \mathrm{mL}$ and successive PSA $\geq 0.2 \mathrm{ng} / \mathrm{mL} .{ }^{17,19,20}$ A secondary analysis tested 3 other definitions for BCR: any PSA $\geq 0.2 \mathrm{ng} / \mathrm{mL}^{6}$ any PSA $\geq 0.4 \mathrm{ng} /$ $\mathrm{mL} ;{ }^{18}$ and any PSA $\geq 0.4 \mathrm{ng} / \mathrm{mL}$ and rising. ${ }^{17}$

Possible predictors of time to BCR can be: baseline patient characteristics (age); pathology (Gleason score, prostate volume, pathologic stage, margin status, perineural invasion); and PSA related criteria (PSA value prior to surgery, time from RP to first detectable PSA, first detectable PSA value).

For each definition of failure, there are events which compete with the event of interest (i.e., BCR). Thus, the statistical techniques are specific to competing risk analysis. The probability of failure was calculated based on cumulative incidence function. ${ }^{21}$ The probabilities of failure between groups of patients were compared using Gray's test and the model was based on Fine and Gray's work.22,23

The time to failure was calculated from the first detectable PSA to the first event (BCR, treatment or last follow-up). The commencement of treatment was considered competing risk event. The covariates considered in the analysis were: age, pathologic T category, prostate volume, percent of tumour, margins, extra-prostatic extension, seminal vesicle invasion, the absolute value of the first PSA detected. The duration between RP and the first detectable PSA and the Gleason score at the time of RP were also analyzed. Lympho-vascular invasion and perineural invasion have more than $10 \%$ missing values (19\% and $21 \%$, respectively) and were not considered in the analysis.

To test for clinical predictors that are associated with rate of PSA rise (i.e., the slope), we employed a mixed effect model. A mixed model is a statistical model containing both fixed effects and random effects. These models are particularly useful in settings where repeated measurements are made on the same statistical units. In our case, we used a mixed effect model to account for the repeated measurement over time of PSA. Similar clinical factors were considered as applied in the former analysis. The outcome was the PSA value and time was measured in months.

\section{Results}

Our cohort consisted of 376 patients (Table 1), with a median age at RP of 61 (interquartile range [IQR] 56-66). The median time from RP to last follow-up in patients who did not receive early salvage treatment was 60.5 months (IQR 40.8-91.5) and the median time from RP to the first detectable PSA was 26.9 months (IQR 11.7-43.0). Based on the D'Amico classification system, 77 (20.5\%) men had lowrisk cancer, $253(67.3 \%)$ men had intermediate-risk cancer and $46(12.2 \%)$ men had high-risk cancer.

The median follow-up time from the first detectable PSA to the end of follow-up among those who did not reach BCR was 18 months (IQR $11-32)$. Only $45.74 \%(n=172)$ of our cohort had a single PSA value of $\geq 0.2 \mathrm{ng} / \mathrm{mL}$, while $15.16 \%$ $(n=57)$ reached the PSA level of $\geq 0.4 \mathrm{ng} / \mathrm{mL}$ and rising.

Despite excluding all subjects receiving adjuvant treatment or not reaching undetectable PSA, 56.4\% $(n=212)$ received salvage treatment prior to the end of follow-up. Of this group, 187 (88.2\%) men received radiation therapy and 25 were treated with ADT (11.8\%). The median PSA value before salvage treatment was $0.16 \mathrm{ng} / \mathrm{mL}$ (IQR 0.11-0.25), with a median follow-up of 6.2 months (IQR 2.8-12.9).

\section{Estimates of time to biochemical recurrence}

Figure 1 illustrates the cumulative incidence of $B C R$, using the competing-risk approach. After 3 years of follow-up, only $50.3 \%$ of patients reached a PSA value above $0.2 \mathrm{ng} / \mathrm{mL}$ (Fig. 1, part A) and only $31.8 \%$ a PSA value $\geq 0.2 \mathrm{ng} / \mathrm{mL}$, and successive PSA $\geq 0.2 \mathrm{ng} / \mathrm{mL}$ (Fig. 1, part B).

Cumulative incidence curves for BCR (defined as an initial PSA $\geq 0.2 \mathrm{ng} / \mathrm{mL}$ and successive PSA $\geq 0.2 \mathrm{ng} / \mathrm{mL}$ ) stratified based on Gleason score (A), pathological stage (B), time from RP to first detectable PSA (C) and value of 
de Boo et al.

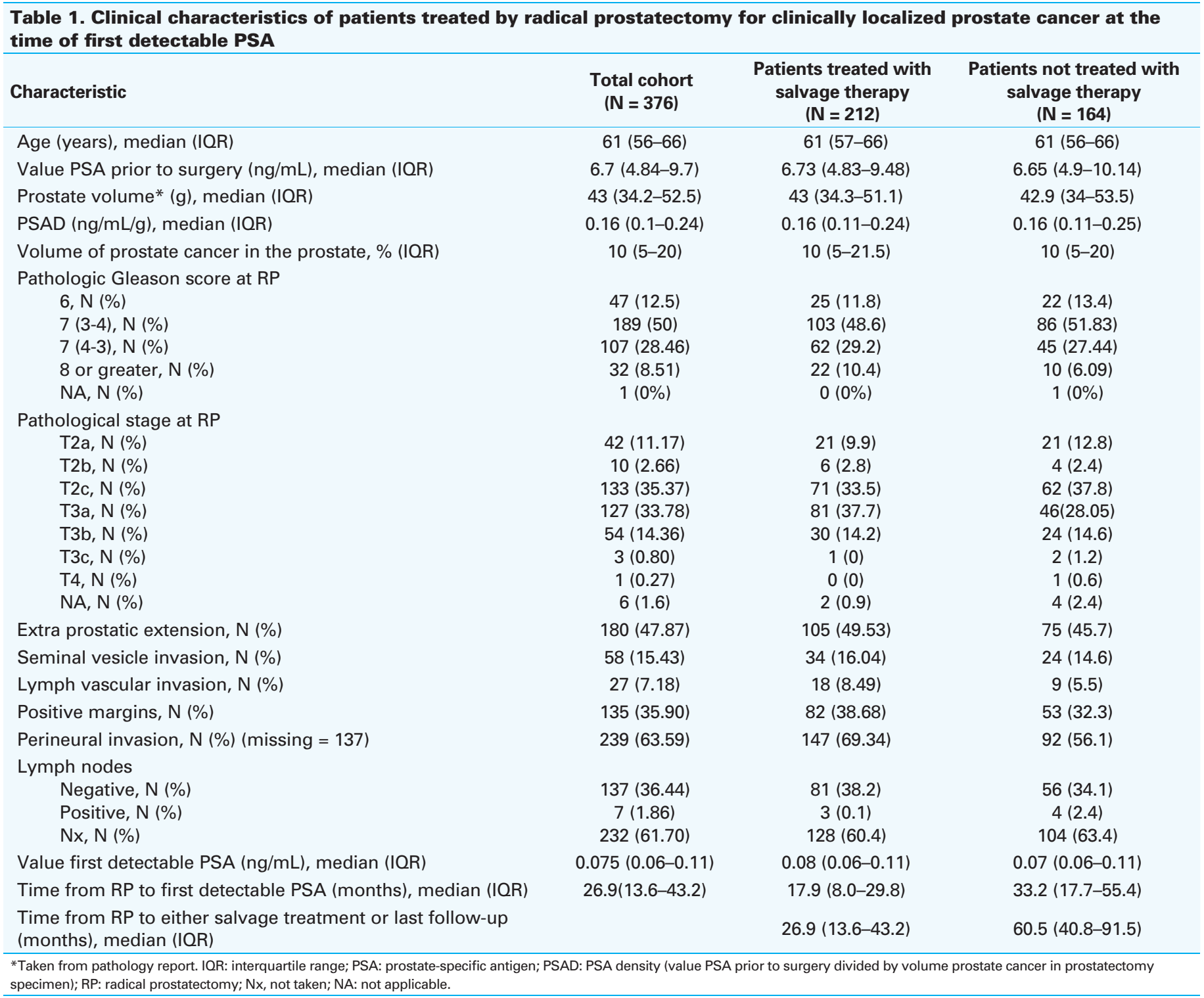

first detectable PSA (D) are depicted in Figure 2. As shown, on univariable analysis a high initial PSA value (higher than 0.1 ), a Gleason score above 8 on RP, and a pathological stage above T3b were associated with earlier time to BCR. Margin status was not associated with BCR. Similar results were reached for the other $\mathrm{BCR}$ definitions.

\section{Predictors of $B C R$}

On multivariable analysis 2 variables were consistently independent predictors of time to reaching any of the 4 BCR definitions (Table 2). The absolute value of the first detectable PSA, as well as Gleason grade 8 or higher, were associated with an increased risk of time to any BCR. Age, PSA value prior to surgery, prostate volume, pathological stage, margin status, and perineural invasion were not associated with time to BCR.

\section{Predictors of rate of PSA rise}

In the mixed effect model we demonstrated that PSA rise was associated with: time from RP to first detectable PSA, Gleason score at RP, and prostate volume. Furthermore, there is an equation that may be used to generate a projected PSA curve for patients with a detectable PSA following RP (Table 3).

In Figure 3 we provide an example of how physicians may use the above mentioned equation and provide a personalized projected slope of PSA rise. In Figure 3, part A the predicted PSA values stratified by Gleason score are plotted 


\begin{tabular}{|c|c|c|}
\hline Predictor & $\begin{array}{l}\text { Univariable analysis } \\
\text { HR (95\% CI) }\end{array}$ & $\begin{array}{l}\text { Multivariable analysis } \\
\text { HR }(95 \% \mathrm{CI})\end{array}$ \\
\hline Age (years) & $1(0.97-1.03), p=0.85$ & \\
\hline Value PSA prior to surgery ( $\mathrm{ng} / \mathrm{mL}$ ) & $1.01(0.98-1.03), p=0.63$ & \\
\hline $\begin{array}{l}\text { Time from RP to first detectable PSA } \\
\text { (months) }\end{array}$ & $1(1-1.01), p=0.17$ & \\
\hline Value first detectable PSA (ng/mL) & $2.06(1.72-2.47), p<0.0001$ & $2.02(1.71-2.39), p<0.0001$ \\
\hline $\begin{array}{l}\text { Pathology Gleason score } \\
\begin{array}{l}6 \text { (ref) } \\
7 \\
8 \text { or greater }\end{array}\end{array}$ & $\begin{array}{c}1 \\
1.95(0.96-3.95), p=0.065 \\
2.43(1.02-5.81), p=0.046\end{array}$ & $\begin{array}{c}1 \\
1.92(0.94-3.91), p=0.071 \\
2.41(1.01-5.79), p=0.049\end{array}$ \\
\hline Prostate volume (g) & $1(0.99-1.01), p=0.85$ & \\
\hline $\begin{array}{l}\text { Pathological stage } \\
\text { T2 and T3a (ref) } \\
\text { T3b/c, T4 }\end{array}$ & $\begin{array}{c}1 \\
1.61(1.05-2.5), p=0.03\end{array}$ & \\
\hline Margins (positive) & $0.96(0.65-1.4), p=0.82$ & \\
\hline Perineural invasion (positive) & $0.84(0.52-1.36), p=0.48$ & \\
\hline
\end{tabular}

\section{Table 3. Equation to generate a projected PSA curve for patients with a detectable PSA following RP \\ $P S A=\exp (A+0.0009 \times R P$ to detection $+0.00018 \times$ volume $+B \times$ time [months] $-0.0003 \times$ time $\times$ RP to detection $-0.00015 \times$ time $\times$ volume) \\ The value of RP to detection is measured in months, the volume is measured in grams, and the time is measured in months. A: 1.94 for Gleason 5 and 6; 1.95 for Gleason 7; and \\ 1.92 for Gleason 8 or more. B: 0.031 for Gleason 5 and 6; 0.056 for Gleason 7; and 0.075 for Gleason 8 or more.}

for a patient who had a first detectable PSA 24 months after surgery and had a prostate volume of $50 \mathrm{~g}$. Figure 3, part $B$ depicts the predicted PSA values stratified by prostate volume for a patient with a Gleason of 7 and a first detectable PSA at 24 months. Similarly in Figure 3, part C the predicted PSA values stratified by time between surgery and first detectable PSA are plotted for a patient with a Gleason 7 and prostate volume of $50 \mathrm{~g}$.

\section{Discussion}

Prior studies demonstrated that BCR after surgery predicts an ominous finding of distant metastasis. ${ }^{7-9}$ However, it may precede clinical disease by many years. In fact, most patients will not develop any clinical manifestations despite having BCR. ${ }^{6,18,24}$ Evidence suggests that adjuvant radiation therapy should be offered to high-risk patients after RP. ${ }^{10-13}$ Critics to this approach cite that this modality was not compared to early salvage therapy in pivotal studies, as well as that more recent trials have failed to demonstrate a survival benefit. ${ }^{25}$ Notwithstanding the fact that many patients with adverse pathology never manifest BCR, its clinical significance is uncertain, and salvage radiation may cure the disease, most urologists prefer to wait before referring patient for radiation. To the best of our knowledge, this is the first study to report estimated time from first detectable PSA to BCR. We report that during the 60-month (IQR, 41-92) median follow-up, only 111 men (29.25\%) in our cohort had BCR according to the definition recommended by the American Urological Association (AUA) and European Association of Urology (EAU). ${ }^{19}$ Thus our results suggest that, on average, the time for patients who have a detectable PSA to reach BCR may be very long. Furthermore our mixed effect model demonstrates that the rate of PSA rise can be projected based on 3 factors: time from RP to first detectable PSA, Gleason score, and prostate volume.

One of the unique challenges we faced was identifying an appropriate cohort of patients. Unlike other studies testing BCR and its predictors, ${ }^{6-9,17-20}$ we wanted to formulate a personalized strategy for the initiation of salvage therapy for patients with a first detectable PSA. The first problem we encountered trying to move forward was that no data existed to estimate median time to BCR among PSA failures. We therefore selected a cohort of patients who had surgery without neo- or adjuvant therapy. All subjects had to have had an undetectable PSA following surgery followed by at least one detectable PSA. In our analysis we also used the first detectable PSA as the cohort entry date, as opposed to the date of surgery. In doing this, our cohort became unbiased, as it includes all patients with a detectable PSA who would be candidates for a future personalized strategy. We had the best available data to estimate median time to BCR among patients with a detectable PSA following surgery.

The main limitation of this study is fact that many patients received early salvage therapy. A total of 212 men (56.4\%) received either radiation or ADT during follow-up. Of these, 133 men received therapy before reaching a PSA of $0.2 \mathrm{ng} / \mathrm{mL}$; these patients could have reached any BCR. We therefore performed our analyses considering such 
de Boo et al.

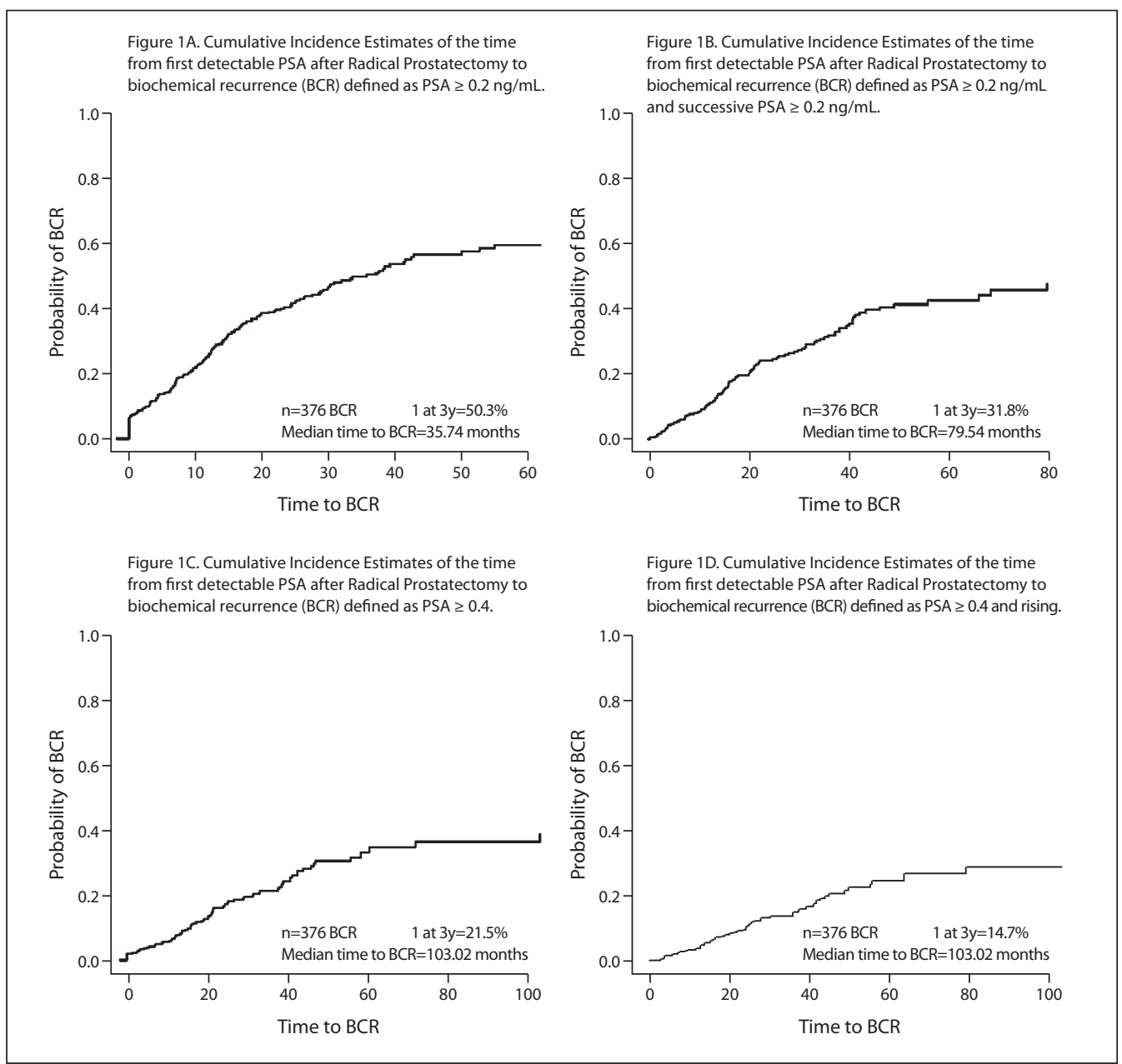

Fig. 1. Cumulative incidence estimates of the time from first detectable $P S A$ after $R P$ to $B C R$ defined as: $A: P S A \geq 0.2 \mathrm{ng} /$ $\mathrm{mL} ; \mathrm{B}$ : PSA $\geq 0.2 \mathrm{ng} / \mathrm{mL}$ and successive PSA $\geq 0.2 \mathrm{ng} / \mathrm{mL} ; \mathrm{C}$ : PSA $\geq 0.4 ; D$ : PSA $\geq 0.4$ and rising. Times indicated are months from first detectable PSA. Estimated cumulative incidence of BCR using the competing-risk approach for all. PSA: prostate-specific antigen; RP: radical prostatectomy; BCR: biochemical recurrence.

events as competing risk events. Our results and estimated median time to BCR reflect current practice among academic urologists who many times offer early salvage therapy to patients even before they reach a PSA of $0.2 \mathrm{ng} / \mathrm{mL}$.

Aside from competing risk analyses, we also assessed PSA slope among patients with detectable PSA post-RP. This analysis was not affected by early salvage therapies. These additional analyses demonstrated that Gleason score, time to first detectable PSA, and prostate volume were independent predictors of the rate of rise. Furthermore we were able to plot a projected PSA rise given individual characteristics. If externally validated, these data may have significant utility in a clinical setting and they can be used to counsel patients on their best time to require salvage radiation based on their disease characteristics. These data are also important as many investigators use patients with detectable PSA to study novel agents (e.g., metformin, statins) that could eventually be used to prevent or delay BCR or augment active surveillance. In some ways these patients may be ideal as, in theory, the PSA production is exclusively secondary to tumour cell production and not confounded by benign prostatic hyperplasia. This study provides a framework by which one can statistically power such a clinical trial.

The effect of prostate size on oncologic outcome after RP remains controversial. ${ }^{26,27}$ Our analysis demonstrated that among patients who have had a detectable PSA following surgery, those who have a smaller prostate will have a higher rise of PSA. The biological mechanism that explains this remains unknown. Smaller prostate size is correlated with higher grade and stage of disease and that may contribute to our findings. ${ }^{26}$ However in our multivariable analysis prostate size was an independent predictor of the rate of PSA increase even after controlling for grade and stage. We hypothesized that more patients with large prostates may have residual benign tissue following RP. This would then contribute to a slower rise in PSA post-surgery. Although the 

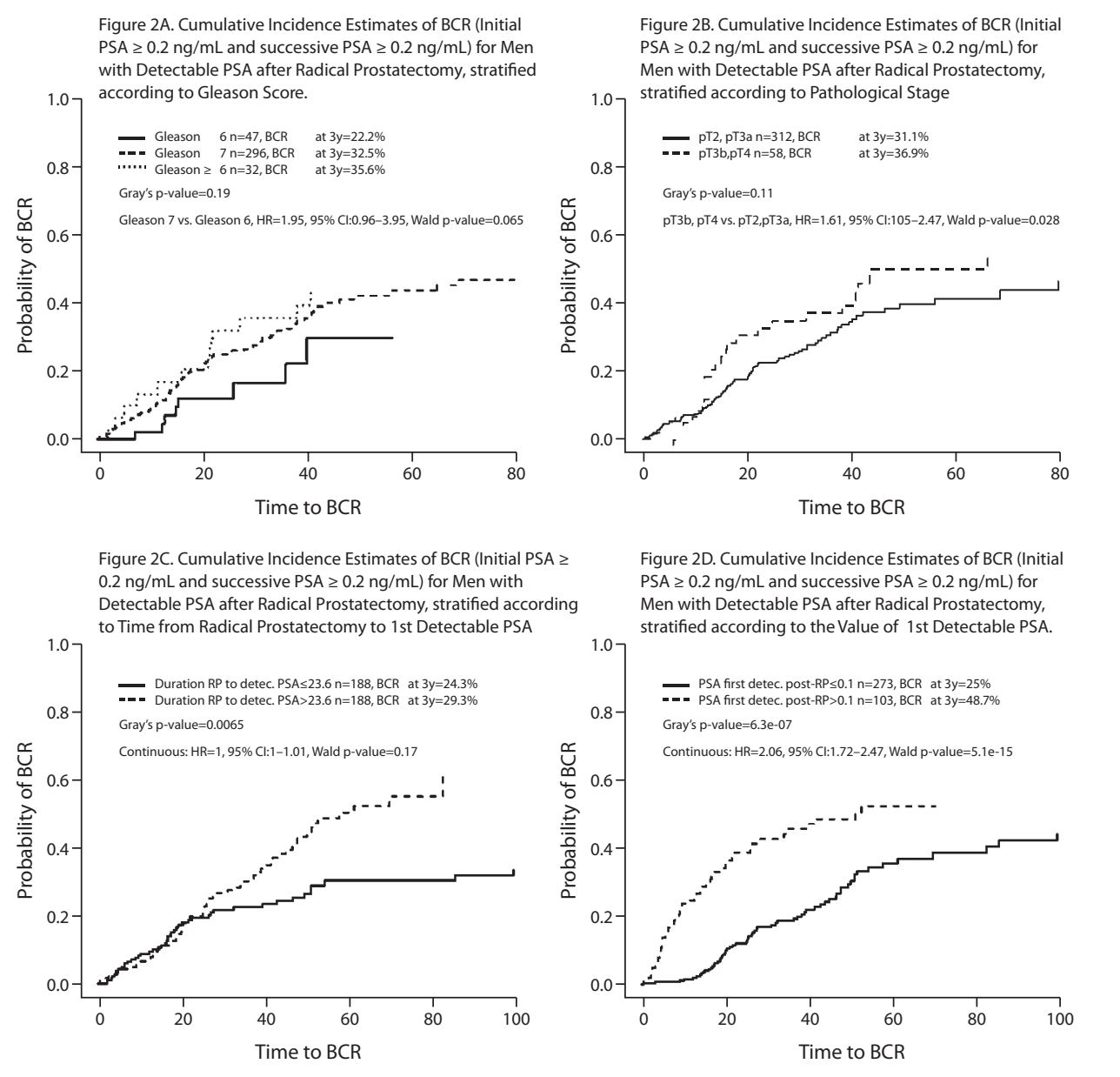

Fig. 2. Cumulative incidence estimates of $B C R$ (initial $P S A \geq 0.2 \mathrm{ng} / \mathrm{mL}$ and successive $P S A \geq 0.2 \mathrm{ng} / \mathrm{mL}$ ) for men with detectable PSA after RP, stratified according to: A: Gleason score; B: Pathological stage; C: Time from RP to first detectable PSA; D: Value of first detectable PSA. Times indicated are months from first detectable PSA. Estimated cumulative incidence of BCR using the competing-risk approach. BCR indicates biochemical recurrence defined as a $P S A$ value $\geq 0.2 \mathrm{ng} / \mathrm{mL}$ and successive PSA $\geq 0.2 \mathrm{ng} / \mathrm{mL}$. PSA: prostate-specific antigen; RP: radical prostatectomy; $B C R$ : biochemical recurrence.

exact biological explanation remains elusive our analysis demonstrated that prostate size was an important predictor of the rate in which PSA will rise among patients with PSA failures.

Another important finding is that margin status was not associated with either time to BCR or rate of PSA rise, contrary to other studies. ${ }^{17}$ The reason for this is that our research question is different than all other studies. We included only patients who had a PSA detected and among these patients we explored predictors of time to reaching BCR, or the rate of PSA rise. Margin status indeed was an important predictor of having a PSA detected after surgery. However for those who already have a PSA failure, it was not associated with a PSA rise.

Our study has several limitations. First, despite the prospective database used, the research question is retrospective. Regrettably, our follow-up time was relatively short.
However, to the best of our knowledge this represents the first systematic analysis of this specific time frame and we believe these results and the associated predictors reported may provide patients and clinicians insight in the natural history of detectable PSA following RP. Second, our results reflect management trends at our tertiary centre and a large proportion of patients received treatment without reaching BCR. It is plausible that shorter estimated median times would have occurred at a centre that does not utilize salvage therapy at such an early state. However, we believe that these results reflect current treatment trends. Lastly our PSA lower detection limit was $0.04 \mathrm{ng} / \mathrm{mL}$; many institutions use ultrasensitive assays for PSA with even lower PSA levels. However these assays would only extend the time to reach $\mathrm{BCR}$, but should not affect the analyses for predictors or the rate of PSA rise. 
de Boo et al.

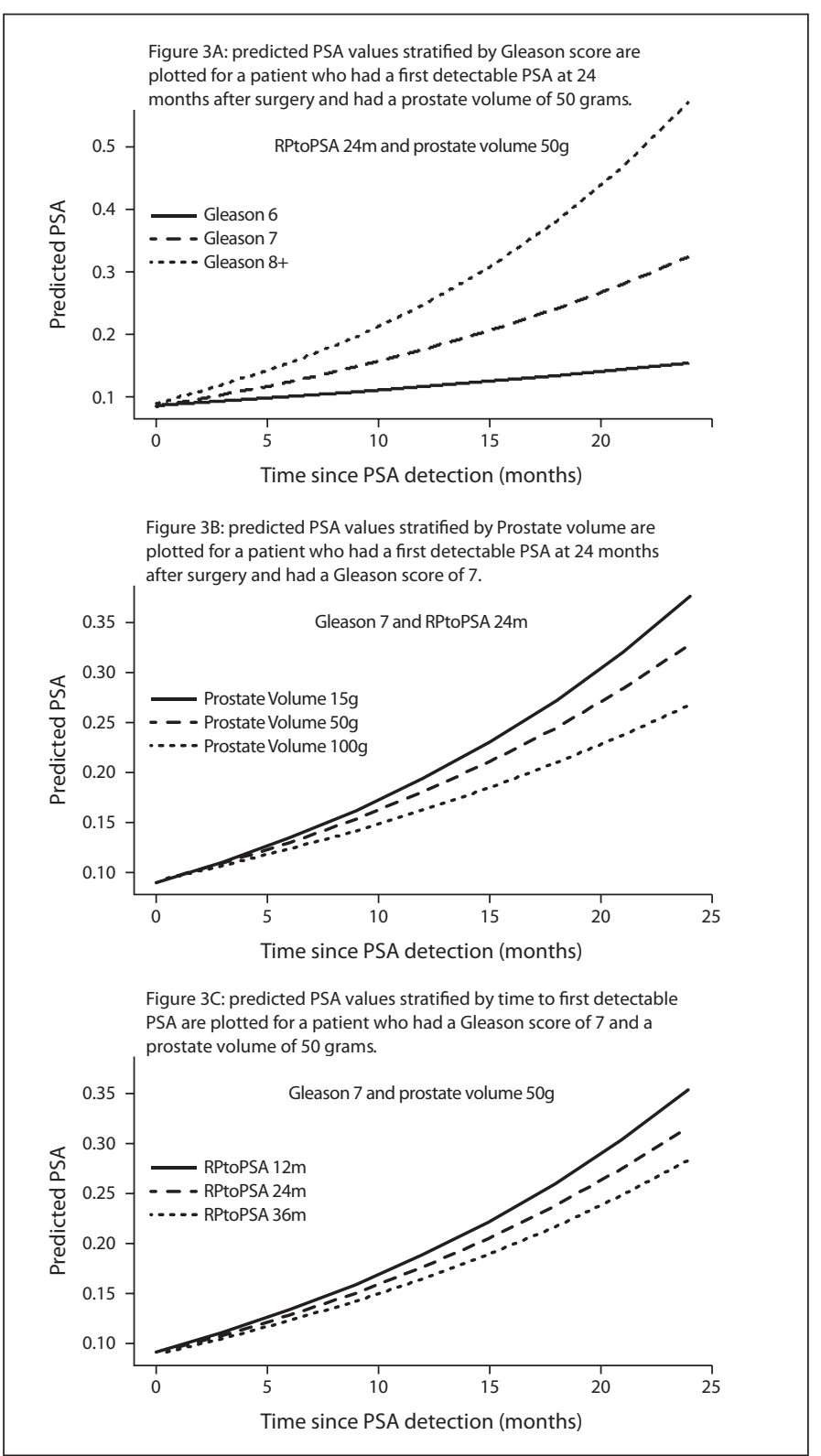

Fig. 3. A: Predicted PSA values stratified by Gleason score are plotted for a patient who had a first detectable PSA at 24 months after surgery and had a prostate volume of $50 \mathrm{~g}$. Here we provide an example of how physicians may use the above mentioned equation and provide a personalized projected slope of PSA rise. In this figure the predicted PSA values stratified by Gleason score are plotted for a patient who had a first detectable PSA at 24 months after surgery and had a prostate volume of $50 \mathrm{~g}$. B: Predicted PSA values stratified by Prostate volume are plotted for a patient who had a first detectable PSA at 24 months after surgery and had a Gleason score of 7 . In this figure the predicted PSA values stratified by Prostate volume are plotted for a patient who had a first detectable PSA at 24 months after surgery and had a Gleason score of 7. C: Predicted PSA values stratified by time to first detectable PSA are plotted for a patient who had a Gleason score of 7 and a prostate volume of $50 \mathrm{~g}$. In this figure the predicted PSA values stratified by time to first detectable PSA are plotted for a patient who had a prostate volume of $50 \mathrm{~g}$ and had a Gleason score of 7. PSA: prostate-specific antigen.

\section{Conclusion}

Our study reveals that the estimated median time from first detectable PSA to BCR may be long. The absolute values of PSA when first detected and Gleason score at RP were independent predictors of the length of this time frame. Furthermore we provided clinicians with an equation that may help them develop a personalized projection of the rate of PSA rise. This equation, if externally validated in a larger multicentre cohort, may be used to counsel patients with a detectable PSA following RP.

Competing interests: Leonora de Boo, Melania Pintilie, Paul Yip, Jack Baniel, and David Margel declare no competing financial or personal interests. Dr. Fleshner is a member of the Advisory Board for Amgen, Janssen, Astellas and Eli Lily. He has received honoraria from Amgen, Janssen, Astellas and Eli Lily. He is and has participated in clinical trials for Amgen, Janssen, Medivation, OICR, and Prostate Cancer Canada.

This paper has been peer-reviewed.

\section{References}

1. Pound CR, Partin AW, Epstein Jl, et al. Prostate-specific antigen after anatomic radical retropubic prostatectomy: Patterns of recurrence and cancer control. Urol Clin North Am 1997;24:395-406. http:// dx.doi.org/10.1016/S0094-0143(05)70386-4

2. Zincke $\mathrm{H}$, Oesterling JE, Blute ML, et al. Long-term (15 years) results after radical prostatectomy for clinically localized (stage T2c or lower) prostate cancer. J Urol 1994;52:1850-7.

3. Trapasso JG, Dekernion JB, Smith RB, et al. The incidence and significance of detectable levels of serum prostate specific antigen after radical prostatectomy. J Urol 1994;152:1821-5.

4. Catalona WJ, Smith DS. 5-Year tumor recurrence rates after anatomical radical retropubic prostatectomy for prostate cancer. J Urol 1994;152:1837-42.

5. Ohori $M$, Goad JR, Wheeler TM, et al. Can radical prostatectomy alter the progression of poorly differentiated prostate cancer? J Urol 1994;152:1843-9. http://jama.jamanetwork.com/article. aspx?articleid=189741

6. Pound CR, Partin AW, Eisenberger MA, et al. Natural history of progression after PSA elevation following radical prostatectomy. JAMA 1999;281:1591-7. http://dx.doi.org/10.1001/jama.281.17.1591

7. Kuriyama $\mathrm{M}$, Wang $\mathrm{MC}$, Lee $\mathrm{Cl}$, et al. Use of human prostate-specific antigen in monitoring prostate cancer. Cancer Res 1981;41:3874-8.

8. Oesterling JE, Chan DW, Epstein Jl, et al. Prostate specific antigen in the preoperative and postoperative evaluation of localized prostatic cancer treated with radical prostatectomy. J Urol 1988;139:766-80.

9. Lange PH, Ercole CJ, Lightner DJ, et al. The value of serum prostate specific antigen determinations before and after radical prostatectomy. J Urol 1989;141:873-7.

10. Thompson IM, Tangen CM, Paradelo J, et al. Adjuvant radiotherapy for pathologically advanced prostate cancer: A randomized clinical trial. JAMA 2006;296:2329-35. http://dx.doi.org/10.1001/ jama.296.19.2329

11. Thompson IM, Tangen CM, Paradelo J, et al. Adjuvant radiotherapy for pathological T3NOMO prostate cancer significantly reduces risk of metastases and improves survival: Long-term follow up of a randomized clinical trial. J Urol 2009;181:956-62. http://dx.doi.org/10.1016/i.juro.2008.11.032

12. Bolla $M$, van Poppel H, Collette L, et al. Postoperative radiotherapy after radical prostatectomy: A randomised controlled trial (EORTC trial 22911). Lancet 2005;366:572-8. http://dx.doi.org/10.1016/ S0140-6736(05)67101-2

13. Wiegel T, Bottke D, Steiner U, et al. Phase III postoperative adjuvant radiotherapy after radical prostatectomy compared with radical prostatectomy alone in pT3 prostate cancer with postoperative undetectable prostate-specific antigen: ARO 96-02/AUO AP 09/95. J Clin Oncol 2009;27:2924-30. http://dx.doi. org/10.1200/JC0.2008.18.9563 
14. Stephenson AJ, Bolla M, Briganti A, et al. Postoperative radiation therapy for pathologically advanced prostate cancer after radical prostatectomy. Eur Urol 2012;61:443-51. http://dx.doi.org/10.1016/i. eururo.2011.10.010

15. Elshaikh MA, Ibrahim DR, Stricker $H$, et al. Adjuvant radiation treatment after prostatectomy. Where do we stand? Can J Urol 2011;18:5592-600.

16. Kibel AS. Treat now or later: the dilemma of postoperative radiotherapy. Eur Urol 2012;61:452-4. http:// dx.doi.org/10.1016/i.eururo.2011.11.044

17. Stephenson AJ, Kattan MW, Eastham JA, et al. Defining biochemical recurrence of prostate cancer after radical prostatectomy: A proposal for a standardized definition. J Clin Oncol 2006;24:3973-8. http:// dx.doi.org/10.1200/JC0.2005.04.0756

18. Amling CL, Bergstralh EJ, Blute ML, et al. Defining prostate specific antigen progression after radical prostatectomy: What is the most appropriate cut point? J Urol 2001;65:1146-51.

19. Mottet N, Bastian PJ, Bellmunt J, et al. Guidelines on prostate cancer. European Association of Urology, April 2014. http://www.uroweb.org/gls/pdf/09\%20Prostate\%20Cancer_LRLV2.pdf. Accessed December $18,2014$.

20. Cookson MS, Aus G, Burnett AL, et al. Variation in the definition of biochemical recurrence in patients treated for localized prostate cancer: The American Urological Association Prostate Guidelines for Localized Prostate Cancer Update Panel report and recommendations for a standard in the reporting of surgical outcomes. J Urol 2007;177:540-5. http://dx.doi.org/10.1016/i.juro.2006.10.097

21. Prentice RL, Kalbfleisch JD, Peterson AV, et al. The analysis of failure times in the presence of competing risks. Biometrics 1978;34:541-54. http://dx.doi.org/10.2307/2530374
22. Gray RJ. A class of K-sample tests for comparing the cumulative incidence of a competing risk. Ann Stat 1988;16:1141-54. http://dx.doi.org/10.1214/aos/1176350951

23. Fine JP, Gray RJ. A proportional hazards model for the subdistribution of a competing risk. JASA 1999;94:496-509. http://dx.doi.org/10.1080/01621459.1999.10474144

24. Freedland SJ, Humphreays EB, Mangold LA, et al. Risk of prostate cancer-specific mortality following biochemical recurrence after radical prostatectomy. JAMA 2005;294:433-9. http://dx.doi.org/10.1001/ jama.294.4.433

25. Bolla $M$, van Poppel H, Tombal B, et al. Postoperative radiotherapy after radical prostatectomy for high-risk prostate cancer: Long-term results of a randomised controlled trial (EORTC trial 22911). Lancet 2012;380:2018-27. http://dx.doi.org/10.1016/S0140-6736(12)61253-7

26. Freedland SJ, Isaacs WB, Platz EA, et al. Prostate size and risk of high-grade, advanced prostate cancer and biochemical progression after radical prostatectomy: A SEARCH database study. I Clin Oncol 2005;23:7546-54. http://dx.doi.org/10.1200/JC0.2005.05.025

27. Davidson DD, Koch MO, Lin $\mathrm{H}$, et al. Does the size matter? Prostate weight does not predict PSA recurrence after radical prostatectomy. Am J Clin Pathol 2010;133:662-8. http://dx.doi.org/10.1309/ AJCPPHGXDI94SGAC

Correspondence: Dr. David Margel, Division of Urology and Davidoff cancer center, Rabin Medical Center, Beilinson Hospital, Israel; sdmargel@gmail.com 